\title{
Patrones de distribución espacio- temporal de los homicidios de mujeres en México en 1990, 2000 y 2010
}

\author{
Patterns of Spatiotemporal Distribution of \\ Femicides in Mexico in 1990, 2000 and 2010
}

Padrões de distribuição espaço-temporais dos homicídios de mulheres no México para 1990, 2000 y 2010

\author{
Francisco Gasca ${ }^{1}$ \\ Escuela de Gobierno y Transformación Pública \\ Tecnológico de Monterrey \\ francisco.gasca@hotmail.com \\ Miguel Flores $^{2}$ \\ Escuela de Gobierno y Transformación Pública \\ Tecnológico de Monterrey \\ miguel.flores@itesm.mx
}

Recibido: $23 / 05 / 2016$

Aprobado: 28/10/2016

1 Doctor en Filosofía con orientación a las Políticas Sociales.

2 Posgrado en Economía y Demografía. 


\title{
Resumen
}

Esta investigación constituye un análisis exploratorio de datos espaciales de los homicidios de mujeres en México a escala municipal. Se realiza un análisis espacio-temporal para los años 1990, 2000 y 2010, con el fin de determinar cambios en los patrones de distribución de los homicidios en mujeres. Con la aplicación de técnicas de estadística espacial y espacio-temporales, se determinó que este fenómeno no se encuentra distribuido aleatoriamente, sino que tiende a concentrarse en determinados espacios. Asimismo, los resultados indican que existe una difusión espacial dirigida hacia el norte del país en el 2010, mientras que en los años 1990 y 2000 había una incidencia mayor en el sur.

Palabras clave: homicidios; datos espaciales; análisis geográfico; municipios.

\begin{abstract}
This research is an exploratory spatial data analysis (ESDA) of femicides in Mexico at the municipal level. This is carried through the application of spatial and spatiotemporal analysis techniques for three different years: 1990, 2000 and 2010. It has the purpose of identify the changes that occur on the femicides patterns. The results suggest that femicides are not randomly distributed but tend to concentrate in certain urban and rural areas. The results also indicate a spatial diffusion towards northern municipalities in 2010; while for 1990 and 2000 a higher incidence was found in southern municipalities.
\end{abstract}

Keywords: femicide; spatial data; geographical analysis; municipalities.

\section{Resumo}

Este artigo deriva-se de uma pesquisa exploratória de dados geográficos dos homicídios de mulheres no México, no nível municipal. Efetua-se uma análise espaço-temporal para os anos 1990, 2000 e 2010, com o objetivo de determinar mudanças nos padrões de distribuição dos homicídios de mulheres. Nesse contexto, com a aplicação de técnicas de estatística espacial e espaço-temporais, determinou-se que este fenômeno não se encontra distribuído aleatoriamente, já que tende a se concentrar em determinadas regiões, tanto urbanas como rurais. Além disso, os resultados indicam que existe uma difusão espacial orientada para as regiões do norte do país no período 2010, enquanto que para os períodos 1990 e 2000, encontra-se uma incidência maior no sul.

Palavras chave: homicídios; dados espaciais; análise geográfica; municípios. 


\section{Introducción}

En el estudio de unidades geográficas es muy difícil pensar que exista independencia espacial entre las mismas, pues es muy probable que lo que ocurra en una unidad territorial repercuta casi directamente en otra, es decir, el valor que tome una variable en una unidad geográfica no solo está determinado por cuestiones internas, sino también por lo que ocurre en las unidades vecinas (Moreno y Vayá, 2000, p. 21).

Lo anterior se relaciona con la primera ley de Tobler (1970), la cual dice que todo tiene relación entre sí, pero que los elementos que se encuentran más cercanos geográficamente se relacionan más entre ellos mismos que entre los que se encuentran más distantes. Esto muchas veces aplica a los estudios de los fenómenos sociales (Vilalta, 2008; Rupasingha y Goetz, 2007; Moreno y Vayá, 2004), donde es muy recurrente encontrar aglomeraciones espaciales en las unidades geográficas de estudio, es decir, que una unidad territorial con determinadas características se encuentre rodeada por otras parecidas, lo que conlleva a una distribución no aleatoria o, dicho de otra manera, que exista dependencia espacial.

En este sentido, el fenómeno de los homicidios y de la violencia en general no escapa de las características de los demás hechos sociales, es decir, igualmente tienden a estar influenciados por lo que ocurre en unidades geográficas vecinas. Particularmente en el contexto mexicano, en el cual en la última década se ha desatado una fuerte escalada de violencia que se han traducido en un incremento en los homicidios, principalmente de mujeres.

De ahí el interés de este trabajo de investigación en conocer los patrones de distribución espacio-temporal de los homicidios de mujeres en México. Aunque ya existen algunos antecedentes del tema de los homicidios en México desde una perspectiva espacial (Valdivia, 2012; Flores y Rodríguez, 2014; Flores y Villarreal, 2015), la revisión de la literatura dio cuenta de que no existe suficiente evidencia empírica que trate este tipo de fenómenos con herramientas de análisis espacial. Por lo que dejar antecedentes sobre el tema es la forma en que se busca aportar a la discusión de los fenómenos con difusión espacial.

Asimismo, es importante destacar que este fenómeno es muy complejo, pues intervienen diversos factores de diferente naturaleza, como de tipo económico, social, cultural, demográfico, geográfico, entre otros, lo que sugiere más estudios desde diferentes campos de la ciencia. Además se le agrega complejidad por la poca disponibilidad de información para determinar si existen razones de género detrás del fenómeno.

De manera particular esta investigación busca responder las siguientes preguntas:

- ¿Los homicidios de mujeres presentan una distribución espacial aleatoria o tiende a concentrarse en determinados espacios para los años 1990, 2000 y 2010 ? Si es así,

3 Cabe hacer mención que para el análisis empírico aquí ofrecido, los homicidios de mujeres se toman como caso particular de violencia de género, de tal forma no se considera la definición legal ni forénsica de feminicidios debido a que la información disponible no cuenta con características en cuanto a la causa y motivación de los homicidios en México. 
- ¿En qué áreas del territorio nacional se concentran significativamente los homicidios de mujeres?

- ¿Existe un patrón de difusión espacio-temporal en los homicidios de mujeres en México?

Estas preguntas conforman el núcleo central de esta investigación y buscan responderse con el análisis de la evidencia empírica.

Este trabajo se encuentra estructurado de la siguiente manera: en el apartado uno, se ofrece una revisión de la literatura sobre la violencia de género y sus factores asociados; enseguida, se realiza un recorrido sobre el análisis exploratorio de datos espaciales abordando sus beneficios, usos y aplicabilidad a diferentes ámbitos de la investigación. En el apartado dos, se detallan los datos que se usaron para esta investigación; en el tres, se explican brevemente las técnicas de análisis espacial que se utilizaron para procesar los datos. En el apartado cuatro se analizan los resultados y, en el quinto, se concluye con algunas reflexiones finales.

\section{Revisión de la literatura}

\subsection{El contexto de la violencia de género y algunos factores asociados}

Aunque en los últimos años parece que la violencia generalizada en México ha ido en aumento, la raíz de este fenómeno no es nueva, pues la historia de México ha estado marcada por sucesos violentos que datan desde períodos de la independencia. Algunos autores han argumentado que la violencia ha sido parte del desarrollo histórico de México, pues desde que el país se hizo independiente se establecieron pautas y patrones culturales de dominación de tipo socioeconómica y racial, las cuales se fueron arraigando en la sociedad mexicana. Hechos históricos, como la guerra contra los Estados Unidos y los franceses y otros conflictos del siglo XIX y del XX como la Revolución, dan muestras claras de la presencia de la violencia en la historia de México (Mestries, 2014).

Asimismo, en épocas modernas parece que los hechos violentos en la sociedad mexicana se han agudizado, particularmente con la llamada guerra contra el narcotráfico iniciada en el 2006, la cual ha enfatizado diversos fenómenos sociales que han llamado la atención de académicos. Dentro de estos fenómenos destaca la violencia de género que en muchos casos se ha traducido a feminicidios (Mora, Reguera, y Calvario, 2014).

La literatura sobre la violencia de género y los factores asociados a ella es relativamente amplia, sin embargo, en el contexto mexicano no es suficiente, dado el incremento de la violencia y de los homicidios en la última década. Fenómenos que han sido foco de atención alrededor del mundo, algunos estudios dan cuenta de ello (Blanco y Villa, 2008; Ávila et al., 2014; Fernández, 2014; Rodríguez, 2014). Estos factores que se asocian a la violencia de género no solo son de tipo socioeconómico, como la pobreza o el bajo nivel de escolaridad, sino también de tipo cultural, como algunos patrones socioculturales de dominación hacia el género femenino, que se encuentran fuertemente arraigados en la sociedad 
mexicana y que se reproducen profundizando las desigualdades que muchas veces se traducen en violencia (Delgado, Estrada \& López, 2015; Vizcarra, 2008).

En este sentido, la cultura patriarcal, que se mezcla con factores de violencia causada por el narcotráfico y con problemas de pobreza, marginación y exclusión, hace que esta mezcla de fenómenos detone con mayor intensidad en el género femenino, como lo han demostrado algunos estudios (Cerva, 2014). Asimismo, existen otros factores que no pueden dejar de mencionarse como el alcoholismo y otras adicciones que han estado asociadas a la violencia de género; existe evidencia empírica que da cuenta de ello (Jaen, Rivera, Amorín y Rivera, 2015).

Mediante estudios empíricos y estadísticas oficiales que miden la cuestión de género, se ha demostrado que el sexo femenino es factor condicionante de vulnerabilidad, empleo precario, y dificultad para acceder al sistema de justicia, a la educación, a la salud, entre otros indicadores de bienestar (González y Galleti, 2015; Vizcarra, 2008; Moctezuma, Narro y Orozco, 2014).

Aunque la literatura relacionada con la violencia de género tiene antecedentes más lejanos, es en la década pasada donde los estudios asociados a este fenómeno han tenido un incremento considerable, principalmente a causa de los feminicidios ocurridos en las zonas fronterizas del norte de México (Aranda, 2014). Muchos actos de violencia hacia la mujer y feminicidios han estado asociados al establecimiento de maquiladoras, principalmente en los estados fronterizos, pues este tipo de establecimientos ocupan mayoritariamente mano de obra femenina por su eficiencia y por su mano de obra barata (Ravelo y Sánchez, 2006; Sánchez, Ravelo y Melgoza, 2015).

Se han documentado casos de violencia a trabajadoras de las maquiladoras y del campo, pues ellas habitan en zonas distantes de sus lugares de trabajo, lo que implica que recorran distancias largas para tomar el transporte público, exponiéndose a situaciones de violencia que en muchos casos se han convertido en feminicidios (Aziz, 2012; Vega, 2012).

Esta situación muchas veces se asocia a condiciones de pobreza, pues en un contexto de violencia, el pertenecer al género femenino y estar en condiciones socioeconómicas precarias, aumenta la condición de vulnerabilidad. La pobreza, vista desde la perspectiva de género, empezó a tener auge a finales de 1980, pues se percibió que este fenómeno tomaba matices y características diferentes en el sector femenino, dado que además de sufrir de condiciones socioeconómicas deficitarias, también padecían otro tipo de dificultades como desigualdad salarial y violencia, tanto en el ámbito privado como en el laboral. Algunos estudios que dan cuenta de la feminización de la pobreza son los de Tortosa (2009) y Tepechín (2011).

En este sentido, se ha reunido evidencia empírica donde se demuestra que la pobreza y los factores asociados a ella, aunado con ciertas condiciones ambientales, como violencia causada por el narcotráfico en algunas zonas del país, y patrones socioculturales que reproducen el machismo, han originado un caldo de cultivo para que el incremento de la violencia hacia las mujeres se haya intensificado en algunos territorios del país (Aranda, 2014).

Es importante señalar que en otros contextos también se han elaborado estudios sobre homicidios de carácter espacial, como el de Garza, Nieto y Gutiérrez (2009) donde se analiza la distribución espacial de los homicidios en una ciudad 
de Colombia, que han detectado concentraciones espaciales significativas. Estudios parecidos son el de Escobar (2012) también en una ciudad de Colombia, el de Formisano (2002) que caracteriza la violencia en Bogotá mediante técnicas de econometría espacial, y el de Moreno (2005) para el caso de Caracas.

Como se mencionó anteriormente, aunque existen algunos antecedentes que abordan la violencia desde la perspectiva espacial en México y en América Latina, la evidencia empírica de los patrones espacio-temporales de los homicidios de mujeres que permitan conocer los cambios en la estructura espacial de este fenómeno, no es suficiente. Así lo muestra la revisión de la literatura, la cual otorga valiosos elementos de análisis que se asocian a la violencia de género, pero muchos de ellos no toman en cuenta el espacio como dimensión donde se desarrollan los fenómenos sociales, incluido el de los feminicidios. De ahí la importancia de conocer las dinámicas espacio-temporales de este fenómeno en el contexto mexicano, mediante el Análisis Exploratorio de Datos Espaciales (AEDE), tema de la siguiente sección.

\subsection{Análisis exploratorio de datos espaciales}

De acuerdo con Anselin (1993), el AEDE puede definirse como el "estudio estadístico de algún fenómeno que se manifiesta así mismo en un espacio como resultado del área, localización o tipología en la cual la interacción espacial llega a ser el foco de atención" (p. 2). A diferencia de otro tipo de análisis estadístico, en el AEDE las observaciones tienen que tener coordenadas geográficas y estas se pueden manifestar en el espacio en forma de puntos, líneas o polígonos.

Igualmente, el AEDE es considerado un conjunto de técnicas que analizan y describen distribuciones espaciales, identifica localizaciones atípicas e identifica patrones espaciales por medio de la detección de clústeres o puntos calientes, es decir teóricamente a esa detección de clústeres se le puede llamar dependencia espacial, mientras que desde el punto de vista técnico puede llamarse autocorrelación espacial (Anselin, 1998, p. 4).

A partir de la década de 1990, el AEDE ha tenido una amplia aplicabilidad en diferentes ámbitos de la investigación, esto a causa de la disponibilidad de los sistemas de información geográfica (SIG) y a la importancia que ha tenido el espacio en los análisis de los diferentes fenómenos sociales. Particularmente con el rescate que hizo Anselin (1995) de los métodos elaborados por Moran (1950) y por las aportaciones a la estadística espacial de Getis y Ord (1992).

Los beneficios de la aplicación de estos métodos de estadística espacial radican en la visualización que se proyecta en forma de mapas, de las variables que dan cuenta de algún fenómeno, pues de esa manera se detectan concentraciones significativas, patrones de distribución y cambios temporales de esos fenómenos objeto de estudio. Esto ayuda a que la Política Pública pueda ser más eficiente cuando se intenta aplicar algún programa, pues por medio de la identificación de clústeres espaciales, se pueden conocer las unidades territoriales en las cuales un fenómeno se presenta con mayor intensidad.

La utilidad y la aplicabilidad del AEDE, mediante la aplicación de técnicas que detectan la autocorrelación espacial, es extensa, pues la evidencia empírica ha demostrado su utilidad en distintos campos de la investigación; por ejemplo, 
existen estudios donde se han determinado patrones de distribución de alguna enfermedad o padecimiento de salud y se han detectado aglomeraciones espaciales significativas que han servido de insumo para la aplicación de políticas públicas en el campo de la salud (Dragicevic y Schuurman, 2004: Owusu y Owens, 2009; Rooney et al., 2014; Szonyi, Srinath, Esteve-Gassent, Lupiani, \& Ivanek, 2015).

Igualmente, el AEDE ha tenido una extensa aplicabilidad en el campo del análisis urbano y de la segregación socio-espacial, pues existen investigaciones que abordan esta temática aplicando este tipo de técnicas, las cuales han dado como resultado estructuras espaciales de las ciudades que dan cuenta de las desigualdades dentro de las mismas; es decir, se pueden observar las unidades territoriales con condiciones deficitarias en algún indicador de bienestar como educación, empleo, vivienda o ingresos. La literatura que aborda estos temas es vasta, particularmente en países latinoamericanos (Álvarez, Lara, Harlow y Denman, 2009; Da Gamma, Marques, Ferreira \& Bitar, 2002; Pérez y Santos, 2011).

El AEDE también ha estado presente en los análisis regionales que tratan sobre los patrones de distribución espacial de la actividad económica; así por ejemplo, se pueden observar investigaciones que tratan el empleo, la productividad, los ingresos, el producto interno bruto y otros indicadores económicos que se aglomeran en determinados espacios, y que pueden reflejar ciertas disparidades regionales (Case, 1991; Le Gallo y Ertur, 2000; Cracolici, Cuffaro y Nijkamp, 2009; Dall'erba, 2005; Ertur y Koch, 2006; Monastiriotis, 2009).

En cuanto a los homicidios y otro tipo de incidentes que ocurren en un espacio geográfico en general, el AEDE también ha sido de utilidad para la detección de concentraciones espaciales significativas de esos fenómenos; existen investigaciones que dan cuenta de las concentraciones de algún tipo de violencia, tanto en el ámbito urbano como en el regional (Messner et al., 1999; Cohen y Tita, 1999; Murray, McGuffog, Western \& Mullins, 2001). Sin embargo, varias de estas investigaciones se han realizado en contextos muy diferentes al latinoamericano, donde las condiciones económicas son menos favorables que en los países desarrollados; igualmente, existen condiciones de marcada desigualdad en muchos indicadores de bienestar y las expresiones de violencia son más intensas que en los países altamente industrializados.

Estas expresiones de violencia toman rasgos particulares en los países latinoamericanos, pues coexisten factores diversos que detonan la violencia de manera singular; por ejemplo, la violencia emergida de las favelas brasileñas propiciadas por las profundas desigualdades sociales en los espacios urbanos, la violencia en Colombia derivada del conflicto armado entre el gobierno y la guerrilla, o el incremento de la violencia en México, que se han traducido en asesinatos particularmente de mujeres, ocasionada por las confrontaciones entre los carteles de la droga.

Este último punto es de lo que trata esta investigación desde una perspectiva espacio-temporal, es decir, se analizan tres puntos en el tiempo, solo los años de 1990, 2000 y 2010, que corresponden a tres décadas diferentes; con esto se pretende examinar los cambios en la distribución de los homicidios de mujeres y así determinar si ha existido un patrón de contracción o difusión de los mismos; a la par, por medio de la generación de mapas, se podrán conocer los municipios donde el fenómeno de los homicidios de mujeres proyecta su mayor intensidad. 


\section{Datos}

La información de los homicidios proviene de las estadísticas vitales del Instituto Nacional de Estadística y Geografía (INEGI). Estos datos consideran todos los tipos de homicidios de mujeres (clasificación internacional de enfermedades, CIE-10: X85-Yo9) que se produjeron durante los años 1990, 2000 y 2010. A pesar de que hemos analizado otras bases de datos que están disponibles actualmente en México, el conjunto final de resultados se basa en datos sobre los homicidios femeninos de las estadísticas vitales oficiales reportados por el INEGI. Esta es la única fuente de información que permite hacer comparables los años antes señalados, además se enfatiza que las fuentes de información no ofrecen elementos para determinar si un homicidio puede considerarse feminicidio, pues las estadísticas oficiales no ofrecen datos sobre el tipo de violencia ni el parentesco de la víctima con el victimario. Sin embargo, se proyectan algunos datos que describen algunas características de los homicidios de mujeres para los tres años de análisis, entre las que se encuentran edad, estado civil y nivel de escolaridad; esto expresado en porcentajes, tal y como se puede observar en la tabla 1.

\begin{tabular}{|c|c|c|c|}
\hline Edad & 1990 & 2000 & 2010 \\
\hline Menores de 1 año & 4,15 & 2,26 & 1,20 \\
\hline 1-4 años & 5,13 & 3,66 & 2,27 \\
\hline 5-9 años & 3,95 & 2,10 & 1,32 \\
\hline 10-14 años & 5,20 & 3,50 & 2,56 \\
\hline 15-19 años & 10,53 & 10,90 & 12,08 \\
\hline 20-24 años & 11,59 & 11,45 & 14,64 \\
\hline 25-29 años & 11,32 & 10,83 & 12,82 \\
\hline 30-34 años & 7,11 & 9,97 & 12,49 \\
\hline 35-39 años & 7,44 & 8,96 & 9,22 \\
\hline 40-44 años & 6,25 & 6,78 & 8,02 \\
\hline 45-49 años & 4,67 & 5,61 & 6,49 \\
\hline 50-54 años & 3,03 & 4,28 & 4,18 \\
\hline $55-59$ años & 3,62 & 3,43 & 2,77 \\
\hline 60-64 años & 3,42 & 3,97 & 2,27 \\
\hline 65-69 años & 2,76 & 2,96 & 1,86 \\
\hline 70-74 años & 1,91 & 2,65 & 1,82 \\
\hline $75-79$ años & 2,37 & 1,71 & 0,95 \\
\hline 80-84 años & 1,84 & 1,56 & 0,54 \\
\hline 85 años y más & 2,17 & 1,95 & 0,79 \\
\hline No especificado & 1,51 & 1,48 & 1,70 \\
\hline
\end{tabular}

Fuente: elaboración de los autores, basado en datos del INEGI. 
En el año 1990, de los homicidios de mujeres reportados, 33\% se concentra entre los 15 y 30 años, situación similar en el año 2000; sin embargo en el año 2010, ese mismo rango de edad concentra casi 40\%, lo que permite deducir que existe cierta intensidad de homicidios de mujeres en edad productiva y joven.

Otro dato que arrojan las estadísticas vitales es el estado civil de estas mujeres; en la tabla 2 , se pueden observar diferentes categorías para los tres años de análisis.

Tabla 2. Estado civil de las mujeres asesinadas para los tres años de análisis (en porcentajes)

\begin{tabular}{lccc}
\hline & $\mathbf{1 9 9 0}$ & $\mathbf{2 0 0 0}$ & $\mathbf{2 0 1 0}$ \\
\hline Menores de 12 años & 14,55 & 8,96 & 5,42 \\
Soltera & 28,51 & 28,97 & 36,39 \\
Casada & 29,89 & 30,92 & 21,63 \\
Unión libre & 9,41 & 12,62 & 14,56 \\
Separada & 0,92 & 1,79 & 0,00 \\
Divorciada & 1,78 & 1,87 & 2,69 \\
Viuda & 8,03 & 8,96 & 5,17 \\
No especificado & 6,91 & 5,92 & 14,14 \\
\hline
\end{tabular}

Fuente: elaboración de los autores, basado en datos del INEGI.

De estas categorías se destaca la concentración de homicidios de mujeres en situación de vida conyugal con 30\% para los años 1990 y 2000, mientras que en el 2010 disminuye a $21 \%$. Aunque las estadísticas vitales no contemplan el parentesco de la víctima y el victimario, estos datos pueden dar indicios de ciertas situaciones de riesgo para las mujeres, pues la literatura menciona que las mujeres no solo sufren violencia en su vida laboral, sino también dentro de su hogar.

Esto contrasta con el resto de las categorías, en las que se muestra un descenso considerable en los porcentajes en las categorías de separada y divorciada, lo que se relaciona con la literatura que aborda cuestiones de violencia de género, en el sentido de que para muchas mujeres el estar separada de su pareja representa menor riesgo de violencia.

Lo anterior se relaciona con el contenido de la tabla 3, pues se puede observar que una cantidad importante de mujeres asesinadas presenta muy baja escolaridad.

La tabla 3 señala que en los tres años de análisis la baja escolaridad representa una situación de riesgo para las mujeres en México, aunque no se puede hablar propiamente de feminicidios, pues las estadísticas vitales no ofrecen información al respecto. Esta información puede ofrecer una guía para asociar la violencia de género con los bajos niveles de escolaridad; asimismo, dicha información se contrasta con el bajo porcentaje de mujeres asesinadas en la categoría de profesional y posgrado. La población en riesgo, o el recuento total de la población de cada municipio, viene a partir de los correspondientes censos de población de 1990, 2000 y 2010. Se enfatiza, que los cálculos se realizaron elaborando un promedio de un año anterior y un año posterior al año señalado; 
posteriormente se estimó una tasa de homicidios de mujeres por cada 100.000 habitantes. Por ejemplo, para calcular la tasa de homicidios para el año 1990, se hizo un promedio para los años 1989, 1990 y 1991; posteriormente se ponderó por la población del censo de 1990. Lo mismo se realizó para los años 2000 y 2010.

Tabla 3. Escolaridad de las mujeres asesinadas para los tres años de análisis (en porcentajes)

\begin{tabular}{lccc}
\hline & $\mathbf{1 9 9 0}$ & $\mathbf{2 0 0 0}$ & $\mathbf{2 0 1 0}$ \\
\hline Sin escolaridad & 18,76 & 13,24 & 10,26 \\
Primaria incompleta & 20,41 & 16,28 & 9,14 \\
Primaria completa & 15,08 & 19,70 & 17,99 \\
Secundaria incompleta & 0,00 & 0,00 & 4,71 \\
Secundaria o equivalente & 11,59 & 16,67 & 19,19 \\
Bachillerato o preparatoria completo & 4,02 & 9,27 & 12,28 \\
Profesional & 4,15 & 6,78 & 8,19 \\
Posgrado & 0,00 & 0,00 & 0,00 \\
No especificada & 26,00 & 18,07 & 18,24 \\
\hline
\end{tabular}

Fuente: elaboración de los autores, basado en datos del INEGI.

Como se muestra en la figura l, las tasas de homicidios de mujeres experimentaron un aumento considerable, sobre todo en 2010 , lo que casi duplicó sus niveles reportados en 2000, 2,57 a 4,21 por cada 100.000 habitantes. Debe tenerse en cuenta que durante esos años, el país fue testigo de un aumento dramático en los niveles de violencia, donde surgieron los escenarios más violentos en zonas con actividades de tráfico de drogas y con presencia de crimen organizado. Sin embargo, la distribución de los homicidios a través de los municipios ha sido mayor en algunas regiones del país; esto puede proyectar patrones de distribución espacial diferentes para cada año.

Figura 1. Tasa de homicidios de mujeres en México para los años 1990, 2000 y 2010 por cada 100.000 habitantes

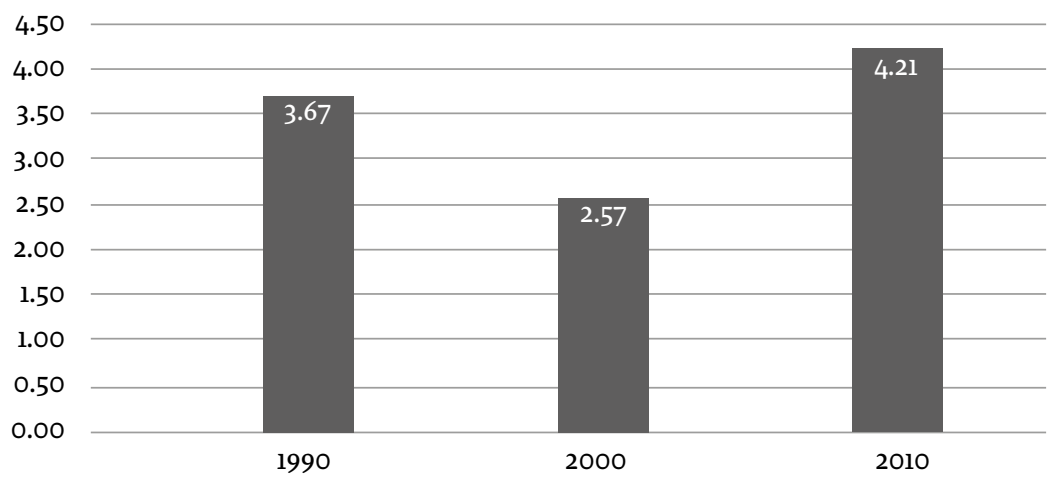

Fuente: elaboración propia, basado en datos del INEGI. 
En la siguiente sección se describen los métodos y software estadístico utilizados en el análisis empírico. El análisis considera entonces métodos estadísticos locales-espaciales y espacio-temporales desde una perspectiva discreta, es decir, las unidades de observación son unidades territoriales con límites geopolíticos, con el objetivo de valorar los patrones de los homicidios de mujeres, a través del espacio y el tiempo. Los métodos descritos se complementan entre sí para una mejor identificación en dos dimensiones, el espacio y el tiempo, con respecto a la distribución de los homicidios de mujeres a escala municipal en México.

\section{Método}

\subsection{Análisis exploratorio de datos espaciales}

Dada la riqueza de los datos en términos de la ubicación precisa de las unidades territoriales y la cobertura de toda la geografía en consideración, el uso de técnicas estadísticas espaciales resulta adecuado en el presente estudio. Aquí resulta conveniente distinguir entre espacio discreto frente a espacio continuo y las técnicas estadísticas adecuadas en ambos casos. El espacio discreto requiere el uso de técnicas de análisis espacial particulares, pues las unidades de observación son unidades administrativas delimitadas territorialmente, que en este caso corresponden a los municipios, mientras que en el espacio continuo las técnicas de análisis espacial son distintas (interpolación, densidad o funciones $K$, por ejemplo), pues en el espacio continuo generalmente las unidades de observación son puntos (Feser y Sweeney, 2002).

Dentro de un marco de espacio discreto, las estadísticas globales frente a las estadísticas locales también pueden diferenciarse. Aunque los indicadores globales como el $I$ de Moran o $C$ de Geary dan información acerca de las tendencias generales de la agrupación, no proporcionan información sobre dónde se encuentran las aglomeraciones. Los indicadores locales, a su vez, permiten la identificación y visualización de los patrones locales de asociación (puntos calientes) y una asignación para inestabilidades locales de asociación espacial global (Anselin, 1995). Entre estos indicadores, el Gettis-Ord $G$ * y los indicadores locales de autocorrelación espacial (LISA, por sus siglas en inglés) son los más utilizados en los estudios empíricos.

Un indicador global común de autocorrelación espacial es la I de Moran global, la cual indica el grado de aglomeración de nuestra variable de interés, en otras palabras, indica si nuestra variable se encuentra distribuida de forma aleatoria a través de los municipios mediante la formulación de una hipótesis nula de aleatoriedad en el total de los datos (Anselin, 1995). La I de Moran global se expresa en la siguiente ecuación:

$$
I=\frac{N \sum_{i j}^{N} w_{i j}\left(x_{i}-\bar{x}\right)\left(x_{j}-\bar{x}\right)}{S_{0} \sum_{i j}^{N}\left(x_{j}-\bar{x}\right)^{2}} i \neq j
$$


donde $N$ es el número de casos, $x$ es la media de la variable, $x_{i}$ es el valor de la variable en una localización particular, $x_{i}$ es el valor de la variable en otro lugar, y $w_{i j}$ es una matriz espacial de peso especificando la interdependencia espacial de $i$ en relación con $j$, y $S_{o}=\Sigma_{i} \Sigma_{j} w_{i j}$. Los valores positivos de este estadístico indican agrupación espacial (por ejemplo, altas tasas de homicidios de un municipio que se encuentran rodeadas por vecinos igualmente con tasas altas), mientras que un valor por debajo de cero indica una autocorrelación espacial negativa, es decir, territorios con valores positivos rodeados de territorios con valores negativos.

Hay que tomar en cuenta que este estadístico sigue una distribución normal asintótica para un $N$ suficientemente grande, de manera que:

$$
Z(I)=\frac{I-E(I)}{[V(I)]^{\frac{1}{2}}} \sim N(0,1)
$$

donde $E(I)$ y $V(I)$ son el valor esperado y la varianza de $I$, respectivamente. La magnitud de $I$ está influenciada por la especificación de la relación espacial entre la unidad de análisis o, en otras palabras, por la elección de la matriz de pesos espaciales $(W)$. En este estudio, los vecinos estandarizados de primer orden por contigüidad tipo Queen se utilizaron como definición de los vecinos. Este análisis exploratorio se realizó con el ArcGis 10.1.

Aunque las medidas espaciales globales ayudan a evaluar la fuerza de autocorrelación espacial en todas las unidades espaciales, generando una estadística global, también pueden existir variaciones espaciales locales en el grado de dependencia espacial. Este último puede ser abordado a través del cálculo de medidas locales de autocorrelación espacial. El uso de las estadísticas locales nos puede informar acerca de la no estacionariedad espacial (varianza heterogénea en las diferentes unidades de estudio) o distintas relaciones espaciales en nuestra variable de interés, identificando así las agrupaciones estadísticamente significativas (Fotheringham, 2009).

Con el fin de analizar la naturaleza de la distribución local de los homicidios, se emplea una versión local de I de Moran, los LISA. Este estadístico evalúa una hipótesis nula de aleatoriedad espacial mediante la comparación de los valores de pares locales, es decir, los valores de cada ubicación específica con los valores en lugares vecinos (Anselin, 1995). Denotada de la siguiente manera:

$$
I_{i}=\frac{z_{i}}{\sum_{i} z^{2} / N} \sum_{i \in I_{i}} w_{i j} z_{j}
$$

donde $Z_{i}$ es el valor correspondiente de la observación $i$ de una variable normalizada particular y $J_{i}$ es el conjunto de observaciones de los vecinos $i$. Teniendo en cuenta las hipótesis de una distribución aleatoria, el valor esperado del estadístico LISA es el siguiente:

$$
E_{A}=-\frac{w_{i}}{N-1}
$$


donde $W_{i}$ es la suma de todos los elementos de la fila que corresponde a la observación $i, \Sigma_{i} w_{i}$.

El estadístico LISA es particularmente útil ya que permite la descomposición de la asociación espacial global en cuatro categorías: cuando un lugar con un valor superior a la media está rodeado de vecinos cuyos valores están también por encima de la media (alto-alto, $\mathrm{HH}$ ) o cuando un lugar con un valor inferior a la media, está rodeado de vecinos con valores igualmente inferiores a la media (bajo-bajo, LL). También se puede dar el caso de que un lugar con un valor superior a la media esté rodeado de vecinos cuyo valor se encuentre por debajo de la media (de mayor a menor, HL), y viceversa (bajo-alto, LH); ver Anselin (1993) para una descripción detallada de las propiedades estadísticas de los LISA.

\subsection{Métodos espacio-temporales}

Hay varias razones para creer que la alta incidencia de los homicidios, no solo se asocie en el espacio, sino que también pueda estar asociado en el tiempo. Por ejemplo, una alta incidencia de la violencia en un lugar puede difundirse a través de las comunidades de vecinos en un proceso similar al contagio (Huesmann, 2011). Por otro lado, una alta incidencia en un período determinado de tiempo puede influir en su incidencia en el próximo período. Ambos efectos, y dado el contexto de este estudio, pueden ser contingentes a las características sociales locales.

El análisis espacio-temporal se lleva a cabo a través de una estadística de exploración del espacio-tiempo retrospectivo basado en el modelo de Poisson discreto. Al hacer esto, grupos de alto riesgo espacio-temporales de los casos de homicidios en los municipios mexicanos se identifican de acuerdo con la población en riesgo subyacente. El análisis de agrupamiento espacio-temporal se llevó a cabo mediante el uso de software de SaTScan.

El SaTScan se ha utilizado en muchos estudios de salud como instrumento de vigilancia para explorar conglomerados de enfermedad en el espacio, el tiempo y el espacio-tiempo, así como en un número considerable de estudios con el objetivo de explorar las agrupaciones del crimen, incluyendo conglomerados espacio-temporales de homicidios en diferentes países (Kulldorff, 2011). El número esperado de casos en cada municipio se calcula entonces como:

$$
E[h]=p * H / P
$$

donde $h$ es el número observado de homicidios, $p$ es la población de la sección censal de interés, y $H$ y $P$ son el número total de homicidios y población, respectivamente. Un riesgo relativo de homicidios para cada municipio se calcula dividiendo el número observado de homicidios por el número esperado de los homicidios. La hipótesis alternativa consiste en que existe un riesgo elevado de homicidio dentro de la ventana de exploración en comparación con las otras unidades de análisis. Bajo el supuesto de Poisson, la función de verosimilitud para una ventana específica es proporcional a: 


$$
\left(\frac{h}{E[h]}\right)^{e}\left(\frac{H-h}{H-E[h]}\right)^{c-e} I
$$

donde $H$ es el número total de homicidios, $h$ es el número observado de homicidios dentro de la ventana, y $E[h]$ es el número esperado de homicidios dentro de la ventana bajo la hipótesis nula de que no haya ninguna diferencia. Debido a que el análisis está condicionado a que el número total de casos observados, $H$ - $E[h]$, es el número esperado de casos fuera de la ventana. $I($ )es una función indicadora, con $I()$ = 1 , es cuando la ventana tiene más casos de lo esperado bajo la hipótesis nula y o en caso contrario (Kulldorff, 2011). La prueba de hipótesis se realiza mediante simulaciones Monte Carlo, en la cual un estadístico de prueba se calcula para cada repetición aleatoria, así como para el conjunto de datos reales.

El estadístico de escaneo espacio-tiempo implica la identificación de una ventana cilíndrica, con un círculo que indica una base geográfica, y con la altura de tiempo, donde el primero indica las áreas de agrupamiento subyacentes, y la altura representa el período de tiempo de los posibles grupos (Kulldorff, 2011). Este proceso abarca el área de estudio, la geografía mexicana de los años 1990, 2000 y 2010, y hace posible la detección de aglomeraciones espacio-temporales.

Una relación de probabilidad logarítmica (LLR, log likelihood ratio) se calcula con base en la diferencia de las incidencias dentro y fuera de las ventanas que acabamos de explicar, y una prueba Monte Carlo ayuda a determinar la significación estadística de los grupos identificados. Una ventana de escaneo o exploración con la LLR máxima, fue considerada como el clúster con mayor probabilidad, indicando que era menos probable que haya ocurrido por casualidad. Además de la aglomeración más probable, otras ventanas de exploración donde los valores LLR fueron estadísticamente significativas. Asimismo, fueron identificados como grupos secundarios y fueron ordenados de acuerdo con su estadístico de razón de verosimilitud.

\section{Análisis de resultados}

México es uno de los países más poblados del mundo, con más de 100 millones de habitantes, distribuidos tanto en grandes zonas metropolitanas como en pequeñas localidades rurales; presenta una distribución desigual en sus ingresos, pues dentro de un mismo espacio urbano, contrastan sectores acaudalados con población en condiciones de pobreza. A esta desigualdad socioeconómica, se le añade una aguda violencia en diversos territorios, que en muchas ocasiones se ha traducido en homicidios (González-Pérez, Vega-López, M.G., Cabrera-Pivarall, Vega-López, A. \& Muñoz-de-la-Torre, 2012).

En ese sentido, este apartado muestra los resultados arrojados por las técnicas de estadística espacial aplicadas a los homicidios de mujeres en los municipios de México, con el fin de determinar patrones en su distribución espacio-temporal.

\subsection{Análisis exploratorio de datos espaciales}

De acuerdo con la aplicación de las técnicas de autocorrelación espacial global. El Índice de Moran parece haberse duplicado en el año 2010, pues pasó de 
0,1691 en el año 2000 a 0,3336 en el año 2010. Estos coeficientes pueden relacionarse con los acontecimientos violentos derivados de la lucha contra el narcotráfico en México, pues en la década pasada ocurrió una escalada de violencia que en muchos casos se ha traducido en homicidios. Esto puede observarse en la figura 2.

Figura 2. Índice de Moran de homicidios de mujeres en los años 1990, 2000, 2010

\section{Índice de Moran}

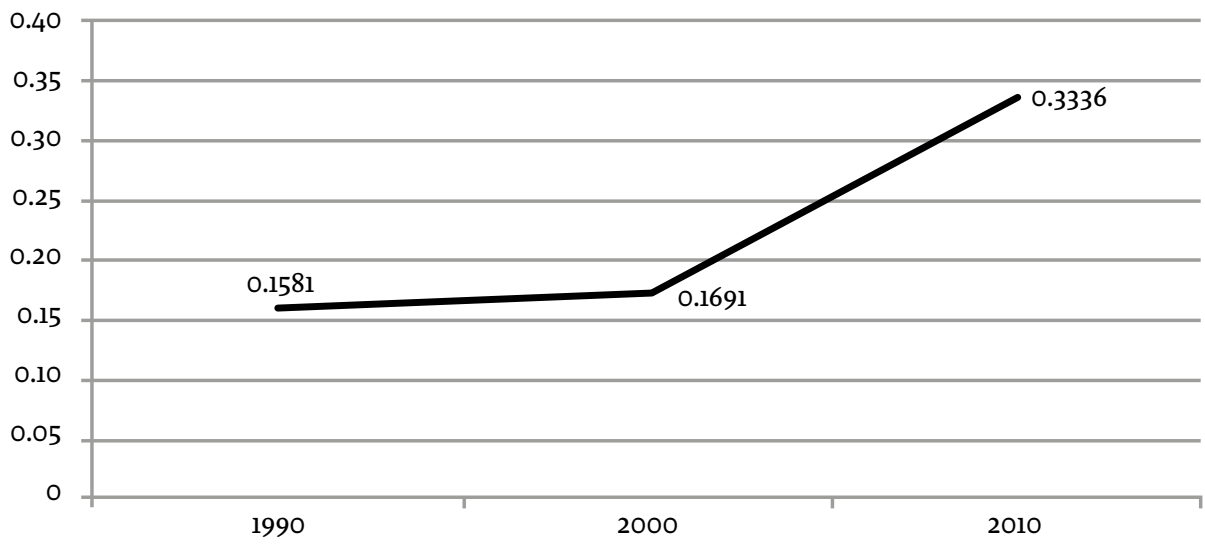

Fuente: elaboración de los autores, basado en datos del INEGI.

De la figura anterior se observa una variación apenas perceptible del estadístico I de Moran, pasando de 0,1581 a 0,1691; un cambio importante resulta cuando se compara con el coeficiente del año 2010. En otros términos, se puede decir que la dependencia espacial de los homicidios en México se ha incrementado en los años de estudio, es decir, la aglomeración espacial se ha hecho más intensa.

Los resultados anteriores señalan un aumento de la autocorrelación espacial global por medio del índice de Moran, sin embargo, no se conocen los territorios donde el fenómeno es más intenso. Para resolver este inconveniente, los indicadores locales de asociación espacial (LISA) ayudarán a detectar aglomeraciones espaciales significativas de los homicidios de mujeres en México.

La tabla 4 muestra los 10 municipios en los que el fenómeno de los homicidios es más intenso, de acuerdo con su índice local, para el año 1990. De esta se observa que la mayoría de los municipios pertenecen al estado de Oaxaca, estado ubicado al sur de México. Asimismo, existen 3 municipios del Estado de México dentro de las primeras 10 posiciones. Esta tabla de posiciones ofrece una idea de la distribución espacial de los homicidios de mujeres en México, pues, de acuerdo con los LISA, el fenómeno parece tener más intensidad al sur y centro del país, principalmente.

Igualmente, es importante destacar que, salvo el municipio de Cuautitlán localizado en el Estado de México, los demás municipios de la tabla 4 presentan cantidades poblacionales menores a 50 mil habitantes; incluso el municipio San 
Martín Itunyoso, ubicado en la posición 1, presenta una población de 2.460 habitantes, lo que habla de pequeños territorios rurales con alta intensidad de dependencia espacial de homicidios de mujeres. Es importante señalar que algunos de estos territorios presentan condiciones de pobreza, particularmente los localizados en el estado de Oaxaca, además, sus unidades económicas son generalmente de baja tecnología, enfocando su actividad económica en pequeños comercios al por menor.

Tabla 4. Clasificación de municipios de acuerdo con su índice local para el año 1990

\begin{tabular}{lllcc}
\hline Posición & \multicolumn{1}{c}{ Estado } & \multicolumn{1}{c}{ Municipio } & Población & Índice local \\
\hline 1 & Oaxaca & San Martín Itunyoso & 2.460 & 118,37 \\
2 & Oaxaca & Putla Villa de Guerrero & 31.897 & 86,41 \\
3 & Oaxaca & Santa María Ipalapa & 4.888 & 59,47 \\
4 & Oaxaca & San Pedro Jicayán & 11.555 & 56,78 \\
5 & Oaxaca & San Lorenzo Texmelúcan & 7.048 & 46,94 \\
6 & Estado de México & Coatepec Harinas & 36.174 & 46,82 \\
7 & Oaxaca & Santo Domingo Teojomulco & 4.571 & 43,71 \\
8 & Estado de México & Cuautitlán & 140.059 & 40,39 \\
9 & Estado de México & Amanalco & 22.868 & 36,59 \\
10 & Oaxaca & San Pedro Atoyac & 4.136 & 35,61 \\
\hline
\end{tabular}

Fuente: elaboración de los autores.

La figura 3 ofrece la localización de los municipios de la tabla 4. En la figura 3, se pueden observar los municipios que presentan aglomeración espacial significativa, es decir, territorios que presentan valores altos en la variable de estudio y que se encuentran rodeados de otros territorios igualmente con valores altos (high-high). También, se pueden observar diversas aglomeraciones espaciales en el norte, centro y sur del país.

Como se dijo anteriormente, los municipios localizados al sur son territorios donde habita una cantidad importante de población rural, y la mayoría de ellos se encuentran en condiciones de pobreza. Esto sugiere que existen elementos de tipo socioeconómico, demográfico y cultural que pudieran intervenir en el fenómeno de los homicidios de mujeres y más concretamente, en la violencia de género.

De acuerdo con estos resultados, en el año 1990, la intensidad de la dependencia espacial de los homicidios de mujeres parece localizarse primordialmente al sur del país, con algunos municipios en el centro. Los resultados del índice de Moran señalan que existe un ligero incremento de la dependencia espacial para el año 2000, por lo que es necesario conocer cuáles fueron los municipios donde el fenómeno de estudio se aglomera significativamente para el año 2000. La respuesta se encuentra en la tabla 5.

La tabla 5 da cuenta de las posiciones de los municipios que presentan mayor intensidad en su autocorrelación espacial, de acuerdo con los homicidios de mujeres. De la misma se pueden observar algunos cambios, pues ahora están presentes algunos municipios del estado de Chihuahua, ubicado al norte del 
país. Esto hace suponer que existe una difusión hacia el norte del país, aunque siguen estando presentes algunos municipios de Oaxaca. En este sentido la tabla 5 da algunos indicios de los patrones espacio-temporales a escala municipal del fenómeno de estudio, pues parece que existe una especie de contagio espacial dirigido hacia el norte.

Figura 3. Mapa LISA de homicidios de mujeres para el año 1990

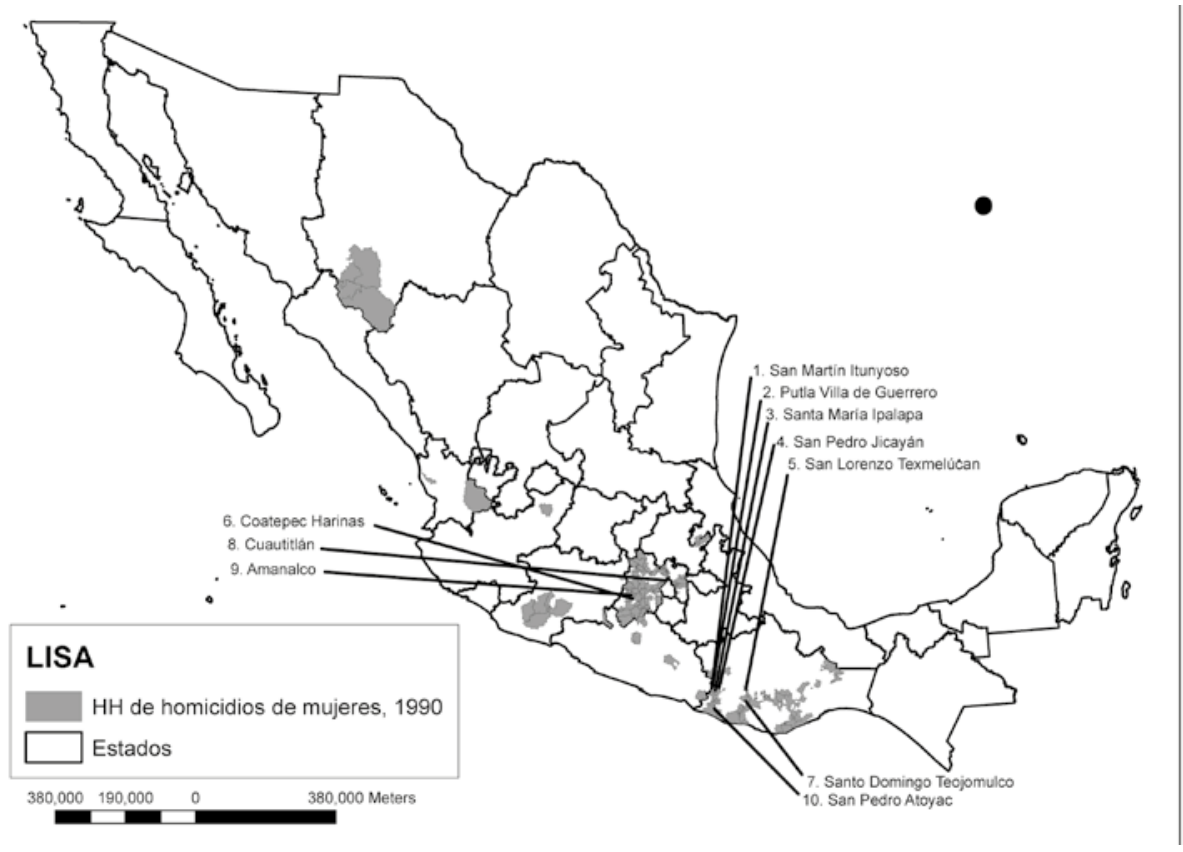

Fuente: elaboración de los autores.

Tabla 5. Clasificación de municipios de acuerdo con su índice local para el año 2000

\begin{tabular}{lllll}
\hline \multicolumn{1}{c}{ Posición } & \multicolumn{1}{c}{ Estado } & \multicolumn{1}{c}{ Municipio } & Población & Índice local \\
\hline 1 & Oaxaca & Santa María Tecomavaca & 1.774 & 140,55 \\
2 & Oaxaca & Santa Lucía Mihuatlán & 3.356 & 134,08 \\
3 & Oaxaca & San Mateo Río Hondo & 3.308 & 111,64 \\
4 & Chihuahua & Batopilas & 14.362 & 109,00 \\
5 & Chihuahua & Morelos & 8.343 & 89,93 \\
6 & Oaxaca & Mazatlán Villa de Flores & 13.435 & 65,44 \\
7 & Oaxaca & Tepelmeme Villa de Morelos & 1.734 & 63,16 \\
8 & Chihuahua & Guachochi & 49.689 & 53,15 \\
9 & Oaxaca & San Juan Bautista Lo de Soto & 2.325 & 52,07 \\
10 & Oaxaca & Santiago Llano Grande & 3.260 & 50,36 \\
\hline
\end{tabular}

Fuente: elaboración de los autores. 
Esto puede observarse en la figura 4, en la cual se aprecian las aglomeraciones espaciales significativas y la presencia de las mismas en los estados del norte del país, incluso se observan algunos municipios fronterizos con valores altos, rodeados de otros municipios igualmente con valores altos. La figura 4 puede contrastarse con la distribución espacial de la figura 3 en la que, para el año 1990, la concentración espacial de homicidios de mujeres tenía mayor presencia en el sur, mientras que para el año 2000 los municipios del norte empezaron a tener mayor presencia.

En la figura 4 también se pueden observar las localizaciones de los municipios de la tabla 5, que corrobora la presencia de los municipios del norte en la intensidad de la autocorrelación espacial en los homicidios de mujeres; estos territorios se encuentran localizados en el estado de Chihuahua.

Figura 4. Mapa LISA de homicidios de mujeres para el año 2000

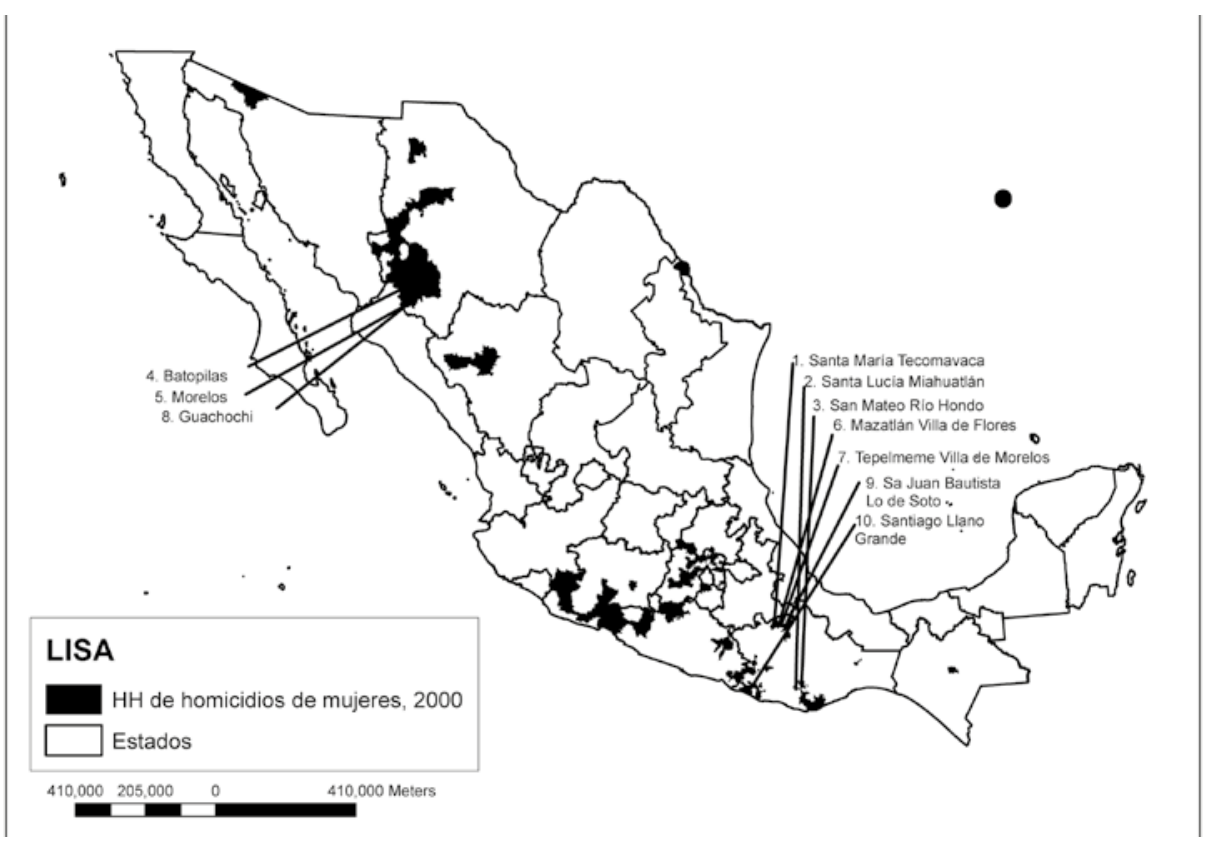

Fuente: elaboración de los autores.

Se destaca igualmente que los municipios de Batopilas, Morelos y Guachochi se encuentran en áreas con fuerte presencia de población indígena (Tarahumaras) y que al igual que los municipios localizados en el sur, presentan condiciones de pobreza. Esto ofrece información sobre las características socioeconómicas de los territorios donde se concentran los homicidios de mujeres. Aunque no ofrece elementos sobre las posibles relaciones entre las condiciones económicas y el fenómeno aquí estudiado, pues no solo existen homicidios de mujeres en territorios pobres, sino también en territorios donde no lo son.

Como se mencionó anteriormente, en el año 2010 el índice de Moran se duplicó en comparación con el año 2000. Esto indica que la magnitud del grado de asociación espacial entre municipios con alta incidencia de homicidios 
aumentó considerablemente y por lo que es de esperarse que hayan ocurrido cambios en los patrones de distribución. Tal como se analizó previamente, para el año 2000, los municipios del norte empezaron a tener mayor presencia en el fenómeno de estudio, por lo que se espera que para el año 2010 esa presencia sea más notoria, así lo hace ver la tabla 6 .

Tabla 6. Clasificación de municipios de acuerdo con su índice local para el año 2010

\begin{tabular}{lllcc}
\hline Posición & Estado & \multicolumn{1}{c}{ Municipio } & Población & Índice local \\
\hline 1 & Chihuahua & Guadalupe & 6.458 & 527,26 \\
2 & Chihuahua & Praxidis G. Guerrero & 4.799 & 343,12 \\
3 & Chihuahua & Juárez & 1.332 .131 & 156,69 \\
4 & Chihuahua & Guachochi & 49.689 & 78,48 \\
5 & Chihuahua & Ojinaga & 26.304 & 60,34 \\
6 & Chihuahua & Urique & 20.386 & 50,74 \\
7 & Chihuahua & Batopilas & 14.362 & 43,35 \\
8 & Chihuahua & Morelos & 8.343 & 39,78 \\
9 & Chihuahua & Uruachi & 8.200 & 33,64 \\
10 & Durango & El Oro & 11.320 & 32,96 \\
\hline
\end{tabular}

Fuente: elaboración de los autores.

En la tabla 6 se puede observar que los 10 municipios con mayor intensidad en la autocorrelación espacial del fenómeno de estudio se encuentran en estados del norte del país, mayoritariamente en Chihuahua. Esto confirma el proceso de difusión espacial hacia el norte del país, que se relaciona con el incremento de la violencia en estados fronterizos del norte, particularmente Chihuahua. Asimismo, estos resultados pueden relacionarse con las aportaciones de investigadores citados en el apartado de revisión de la literatura, pues han arrojado evidencia de algunos factores asociados a la violencia de género, destacando el establecimiento de maquiladoras en los estados fronterizos y la guerra entre carteles de la droga en esos mismos estados.

Retomando los resultados de la tabla 6, es importante destacar la presencia de un importante centro urbano como lo es Juárez, con una población de más de un millón de habitantes. Esto puede relacionarse con lo comentado en el apartado de la revisión de la literatura, pues esa ciudad ha sido foco de atención mundial por el fenómeno de los homicidios. La localización de Juárez y de los otros municipios de la tabla 3 pueden observarse en la figura 5 .

Aunque siguen existiendo aglomeraciones espaciales significativas en el sur del país, es evidente que ha existido un cambio en los patrones de distribución espacial. Esto porque existe mayor presencia en el norte para el último año de análisis, igualmente los municipios que presentan mayor intensidad en la autocorrelación se encuentran en esa región del país, esto para el 2010, a diferencia de la concentración de los años 1990 y 2000, en los cuales la mayor intensidad se presentaba en el sur y centro del territorio nacional. 
Figura 5. Mapa LISA de homicidios de mujeres para el año 2010

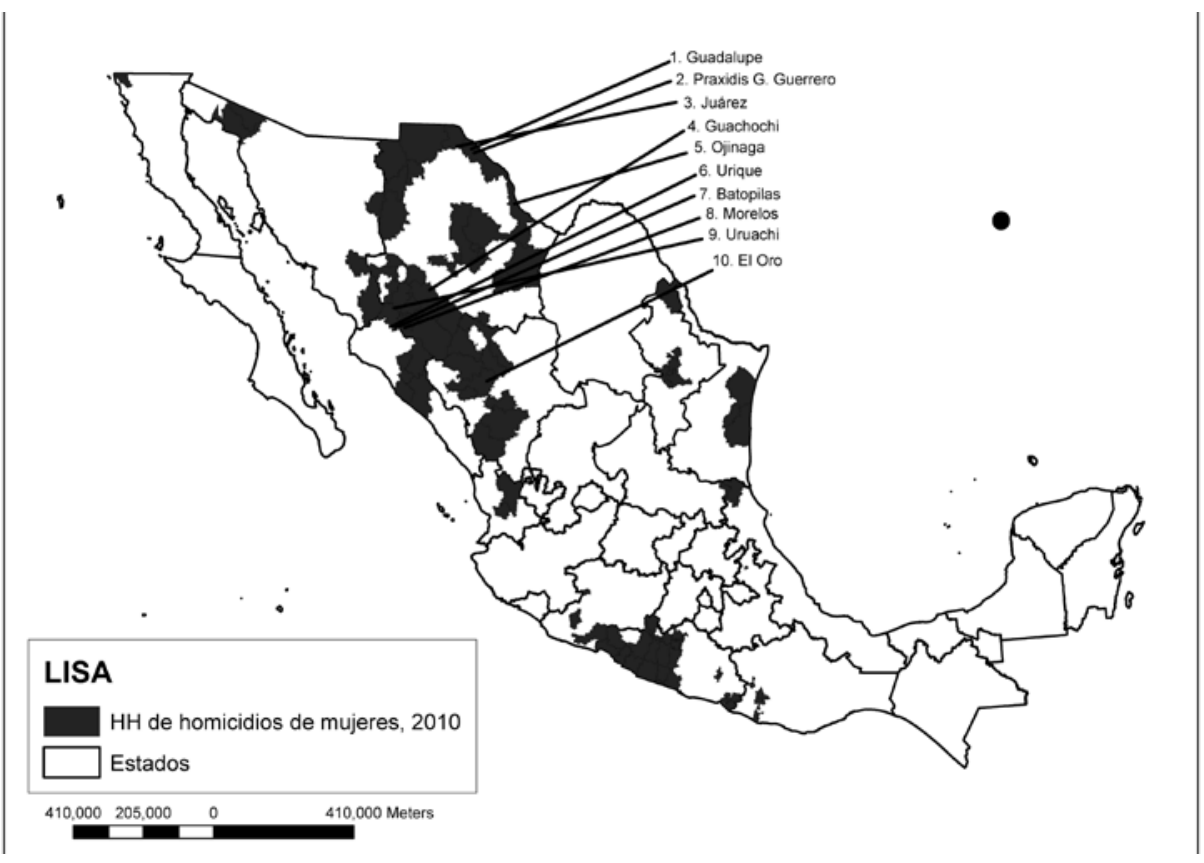

Fuente: elaboración de los autores.

\subsection{Análisis espacio-temporal}

De acuerdo con la aplicación de métodos espacio-temporales con el software SatScan, el cual tomó en cuenta la tasa de homicidios de mujeres a escala municipal en los tres años de estudio, los municipios que presentan mayor aglomeración espacio-temporal se localizan tanto en el sur del país como en el norte. Por ejemplo, se pueden observar municipios localizados en los estados de Chihuahua, Nuevo León y Durango (norte), como municipios localizados en Oaxaca y Guerrero (sur).

La tabla 7 da cuenta de los municipios en los que espacio-temporalmente, los homicidios de mujeres ocurren con mayor intensidad. Puesto que se toman tres años en el tiempo, es de esperarse que aparecieran municipios localizados en diversas partes del país, pues como se analizó previamente, en el año 1990, la ocurrencia de homicidios de mujeres en el país se localizaba mayoritariamente en el sur, mientras que en el 2010 esta intensidad se enfatizaba en los municipios del norte.

Es de destacar que salvo el municipio de Juárez, los demás territorios cuentan con una población relativamente baja, lo que da cuenta de la ruralidad del fenómeno de los homicidios. Por ejemplo, el municipio de Vallecillo, ubicado en el estado de Nuevo León, cuenta con una población de menos de 2 mil habitantes y con un riesgo relativo alto. Los 10 municipios con mayor riesgo relativo se pueden apreciar en la figura 6.

En la figura 6 se pueden observar dos tipos de íconos, triángulos y círculos; los primeros señalan los municipios donde espacio-temporalmente se presenta mayor riesgo relativo del fenómeno de estudio; los segundos pueden interpretarse como 


\begin{tabular}{lllll}
\hline \multicolumn{4}{c}{ Tabla 7. Clasificación de municipios de acuerdo con su riesgo relativo } \\
\hline \multirow{2}{*}{ Posición } & \multicolumn{1}{c}{ Estado } & \multicolumn{1}{c}{ Municipio } & Población & \multicolumn{1}{c}{ Riesgo relativo } \\
1 & Chihuahua & Guadalupe & 6.458 & 87,08 \\
2 & Oaxaca & San Bartolo Coyotepec & 8.684 & 83,92 \\
3 & Nuevo León & Vallecillo & 1.971 & 64,05 \\
4 & Oaxaca & San Pedro Ixtlahuaca & 6.822 & 37,34 \\
5 & Chihuahua & La Cruz & 3.982 & 37,12 \\
6 & Chihuahua & Praxedis G. Guerrero & 4.799 & 32,87 \\
7 & Chihuahua & Juárez & 1.332 .131 & 30,41 \\
8 & Chihuahua & Santa Isabel & 3.937 & 30,38 \\
9 & Guerrero & Coahuayutla & 13.025 & 28,29 \\
10 & Durango & Otáez & 5.208 & 27,96 \\
\hline \multicolumn{5}{r}{ Fuente: elaboración de los autores. } \\
\hline
\end{tabular}

Figura 6. Riesgo relativo de homicidios de mujeres en municipios mexicanos

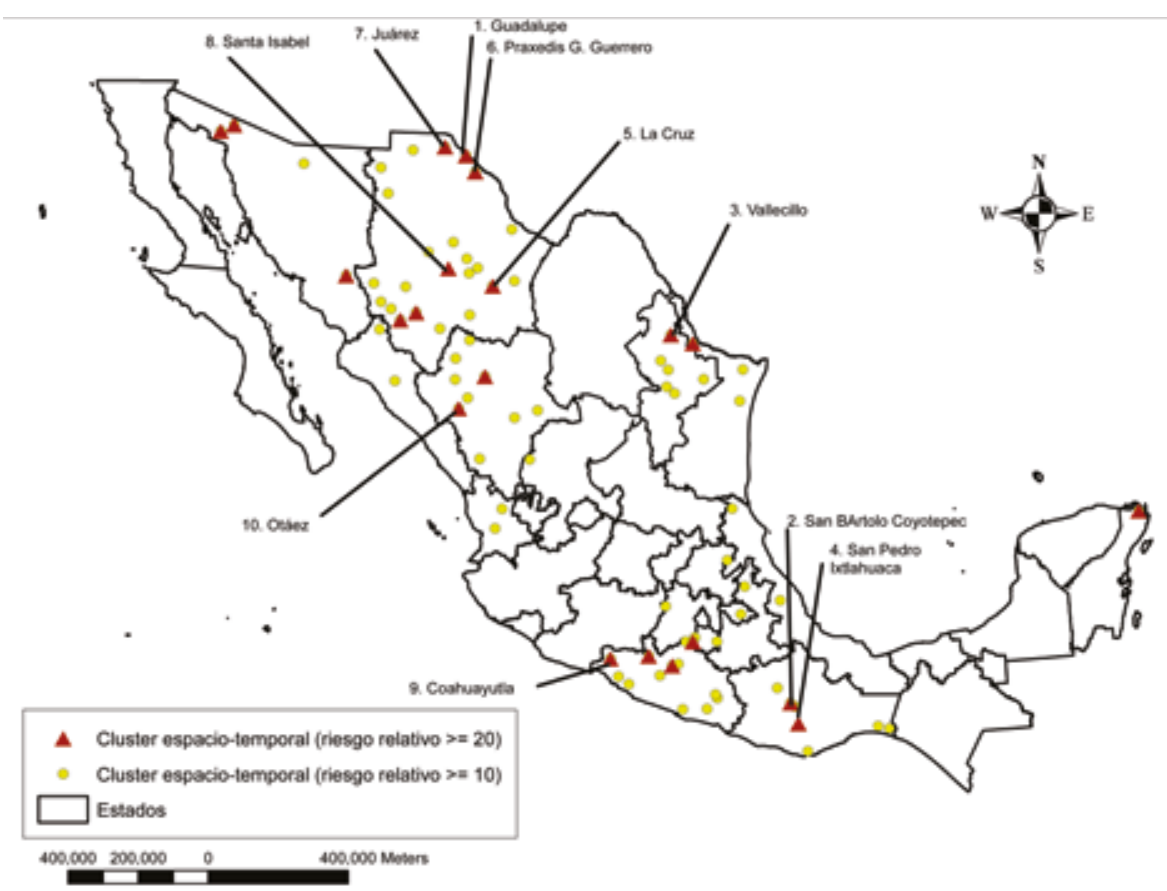

Fuente: elaboración de los autores.

aglomeraciones secundarias, en las que el riesgo relativo es mayor a 10 pero menor a 20. Igualmente pueden observarse los 10 municipios de la tabla 7 con mayor riesgo relativo de homicidios de mujeres en México. 
De estos resultados, se destaca la prevalencia del riesgo en los municipios particularmente localizados en los estados fronterizos, específicamente los localizados en Chihuahua, estado que ha cobrado la atención mundial por el incremento de muertes de mujeres en la década pasada.

Es importante señalar que en estos estados fronterizos se han instalado una gran cantidad de maquiladoras, empresas que se relacionan con muchos feminicidios, pues como se analizó previamente en la revisión de la literatura, muchas de las mujeres que trabajan en esas maquiladoras corren un alto riesgo de sufrir violencia de género derivada de rasgos culturales como el machismo, el cual padecen tanto fuera del hogar como adentro. Muchas de estas empresas se localizan en lugares desérticos, distantes de los lugares habitacionales, y despoblados, lo que expone a muchas trabajadoras a peligros propios de territorios en los que se ha agudizado la violencia derivada por la lucha de los carteles de la droga.

En ese sentido, por el hecho de ser zonas fronterizas, los carteles de la droga luchan por apropiarse tanto del mercado local como del internacional, aunado a la guerra contra el narcotráfico declarada por el Gobierno Federal; estos territorios han sido escenario de diversos fenómenos sociales, como la migración y los homicidios de mujeres, estos últimos tratados en esta investigación desde una perspectiva espacio-temporal.

\section{Conclusiones}

Este estudio ha proporcionado nuevas perspectivas sobre la distribución espacial de los homicidios de mujeres en México. El análisis ha utilizado métodos espaciales y espacio-temporales con el objetivo de identificar, a través de los municipios, las concentraciones significativas de incidencia de homicidios de mujeres durante tres años diferentes, 1990, 2000 y 2010. Los resultados indican que los homicidios de mujeres no presentan una distribución aleatoria, sino que tienden a concentrarse en determinados espacios, con estos resultados queda contestada la primera pregunta de investigación.

Estas concentraciones tienen diferente intensidad de acuerdo con el año analizado; así para 1990, los diez municipios que presentaban mayor intensidad en su correlación se ubicaban en el centro y sur del país. Para el 2000, esta intensidad empezaba a mostrarse en municipios del norte, y finalmente para el 2010, la intensidad se reflejaba mayoritariamente en los estados fronterizos.

Estos cambios en los patrones de distribución se relacionan con los acontecimientos ocurridos en la década pasada, particularmente con la llamada guerra contra el narcotráfico y el aumento de los homicidios de mujeres en los estados del norte del país, temas que han sido abordados ampliamente en la literatura sobre la violencia de género. Estos resultados se relacionan con la segunda pregunta de investigación, en el sentido de que las concentraciones significativas del fenómeno de estudio se localizan en determinados territorios, particularmente en sur y en estados fronterizos del norte.

Asimismo, de acuerdo con los resultados arrojados por aplicación de métodos espacio-temporales, se encontraron aglomeraciones significativas que son persistentes en el tiempo, localizadas en diversos puntos del territorio nacional. Estos resultados corroboran los cambios espacio-temporales de los homicidios de 
mujeres, pues ha existido un patrón de distribución dirigido hacia los municipios del norte del país, por lo que con estos resultados, la tercera pregunta de investigación puede ser contestada, al detectarse un patrón de difusión hacia el norte.

Es importante destacar que muchas de las aglomeraciones espaciales y espacio-temporales se localizan en municipios en condiciones de pobreza y con población indígena, así mismo, se destaca que salvo el municipio de Juárez que cuenta con una población de más de un millón de habitantes y Cuautitlán con más de 140 mil, todos los demás presentan cantidades poblacionales menores a 50 mil habitantes, incluso algunos con menos de 5 mil, lo que señala la ruralidad del fenómeno.

De este modo se puede deducir que la violencia expresada en términos de homicidios de mujeres se encuentra mejor refugiada en territorios que carecen de instituciones que ofrecen protección y seguridad a grupos vulnerables, como lo es muchos municipios rurales del país, donde incluso prevalecen los usos y costumbres en las que el género femenino es un grupo altamente vulnerable.

En este sentido, es necesario formular nuevas interrogantes de investigación que impliquen la relación entre la violencia de género y las características socioeconómicas de la población, particularmente las condiciones de pobreza, el nivel educativo, origen étnico y componente rural-urbano, como se señaló anteriormente. Asimismo, este estudio constituye un análisis exploratorio sobre la estructura espacial de los homicidios de mujeres a escala municipal, lo que sugiere la aplicación de otro tipo de técnicas que permitan mayor inferencia y que involucren los elementos antes mencionados con el fin de arrojar más evidencia empírica sobre el fenómeno de la violencia de género.

\section{Referencias}

Álvarez, G., Lara, F., Harlow, S. y Denman, C. (2009). Mortalidad infantil y marginación urbana: análisis espacial de su relación en una ciudad de tamaño medio del noroeste mexicano. Revista Panamericana de Salud Pública, 26(1), 31-8. Anselin, L. (1993). Exploratory Spatial Data Analysis and Geographic Information Systems. Research Paper 1923. Morgantown (WV), US: West Virginia University. Anselin, L. (1995). Local Indicators of Spatial Association-LISA. Geographical Analysis, 27(2), 93-115.

Anselin, L. (1998). Exploratory Spatial Data Analysis in a Geocomputational Environment. Working Paper. Morgantown, US: Regional Research Institute and Department of Economics West Virginia University.

Aranda, P. (2014). De espacios y violencias: vida cotidiana de jornaleras en comunidades del noroeste de México. Región y Sociedad, (número especial 4), 189-216.

Ávila, L., Valdez, R., Barroso, A., Híjar, M., Rojas, R. y del-Río, A. (2014). Prevalencia y factores asociados a violencia de pareja en usuarias de servicios públicos de salud en México: un análisis comparativo. Revista de Investigación Clínica, 66(1), 45-58.

Aziz, A. (2012). Violencia y destrucción en una periferia urbana. El caso de Ciudad Juárez, México. Gestión y Política Pública, Volumen temático, 227-268. 
Blanco, L. \& Villa, S. (2008). Sources of Crime in the State of Veracruz: The Role of Female Labor Force Participation and Wage Inequality. Feminists Economics, 14(13), 51-75.

Case, A. (1991). Spatial Patterns in Household Demand. Econometrica, 54(4), 953-965.

Cerva, D. (2014). Participación política y violencia de género en México. Nueva Época, LIX(222), 117-140.

Cohen, J. \& Tita, G. (1999). Diffusion in Homicide: Exploring a General Method for Detecting Spatial Diffusion Processes. Journal of Quantitative Criminology, 15(4), 451-493.

Cracolici, M. F., Cuffaro, M. \& Nijkamp, P. (2009). A Spatial Analysis on Italian Unemployment Differences. Statistical Methods and Applications, 18(2), 275- 291.

Dall'erba, S. (2005). Distribution of Regional Income and Regional Funds in Europe 1989-1999: An Exploratory Spatial Data Analysis. Annals of Regional Science, 39(1), 121-148.

Da Gamma, H., Marques, E., Ferreira, M. \& Bitar, S. (2002, Nov.). Poverty and Space: Patterns of Segregation in Sao Paulo. Paper to be presented in the Workshop on Spatial Segregation and Urban Inequality in Latin America. Austin, US.

Delgado, C., Estrada, B. \& López, J. (2015). Gender and Cultural Effects on Perception of Psychological Violence in the Partner. Psicothema, 27(4), 381- 387.

Dragicevic, S. \& Schuurman, N. (2004). The Utility of Exploratory Spatial Data Analysis in the Study of Tuberculosis Incidences in an Urban Canadian Population. Cartographica, 39(2), 29-39.

Ertur, C. \& Koch, W. (2006). Regional Disparities in the European Union and the Enlargement Process: an Exploratory Spatial Data Analysis, 1995-2000. Annals of Regional Science, 40(9), 723-765.

Escobar, G. (2012). El uso de la teoría de la desorganización social para comprender la distribución de homicidios en Bogotá, Colombia. Revista INVI, 27(74), 21-85. Recuperado de: http://revistainvi.uchile.cl/index.php/INVI/article/ view/606/1012

Fernández-de-Juan, T. (2014). La educación sexual y de género vs el maltrato en la pareja. Escenario sobre la violencia en jóvenes de Baja California. Estudios Fronterizos, 15(30), 73-96.

Feser, E. \& Sweeney, S. (2002). Theory, Methods and a Cross-metropolitan Comparison of Business Clustering. In P. McCann (Ed.), Industrial location economics (pp. 222-262). London, UK: Edward Elgar Publishing.

Flores, M. \& Villarreal, A. (2015). Exploring the Spatial Diffusion of Homicides in Mexican Municipalities Through Exploratory Spatial Data Analysis. Cityscape, 17(1), 35-49.

Flores, M. \& Rodríguez, E. (2014). Spillover Effects on Homicides across Mexican Municipalities: A Spatial Regime Model Approach. The Review of Regional Studies, 44(3), 241-262.

Formisano, M. (2002). Econometría espacial: características de la violencia homicida en Bogotá. Documento CEDE, 10. Bogotá, Colombia: Universidad de los Andes.

Fotheringham, S. (2009). The Problem of Spatial Autocorrelation and Local Spatial Statistics. Geographical Analysis, 41(4), 398-403. 
Garza, N. F., Nieto, L. y Gutiérrez, M. (2009). El homicidio en Barranquilla: una lectura espacial. Revista de Economía del Caribe, (3), 176-229.

Getis, A. \& Ord, K. (1992). The Analysis of Spatial Association by Use of Distances Statistics. Geographical Analysis, 24(3), 189-206.

González-Pérez, G. J., Vega-López, M. G., Cabrera-Pivarall, C. E., Vega-López, A. \& Muñoz-de-la-Torre, A. (2012). Deaths by Homicide in Mexico: Trends, Socio-geographical Variations and Associated Factors. Ciência \& Saúde Coletiva, 17(12), 3195-3208.

González, M. y Galletti, G. (2015). Intersecciones entre violencia de género, pobreza y acceso a la justicia: el caso de la Ciudad de La Plata. Oñati Socio-legal, 5(2), 520-546.

Huesmann, R. (2011, April 29). The Contagion of Violence: The Extent, the Processes, and the Outcomes. Presentado en National Academies of Science's Institute of Medicine's Global Forum on Violence. Washington D.C., US. Recuperado de http://rcgd.isr.umich.edu/aggr/articles/Huesmann/20ll.Huesmann.The\%20 Contagion\%20of\%20Violence.NAS\%20Press.pdf

Jaen, C., Rivera, S., Amorín, E. y Rivera, L. (2015). Violencia de pareja en mujeres: prevalencia y factores asociados. Acta de investigación Psicológica, 5(3), 2224- 2239.

Kulldorff, M. (2011). SatScan Software for spatial, temporal and space-time scan statistics (version 9.4.2) [software]. Recuperado de http://www.satscan.org/

Le Gallo, J. \& Ertur, C. (2000). Exploratory Spatial Data Analysis of the Distribution of Regional Per Capita GDP in Europe. 1980-1995. Papers in Regional Science, 82(2), 175-201.

Messner, S., Anselin, L., Baller, R., Hawkings, D., Deane, G. \& Tolnay, S. (1999). The Spatial Patterning of County Homicide Rates: An Application of Exploratory Spatial Data Analysis. Journal of Quantitative Criminology, 15(4), 423-450.

Mestries, F. (2014). La crisis en México y las raíces de la violencia social. El Cotidiano, (187), 59-66.

Moctezuma, D., Narro, J. y Orozco, L. (2014). La mujer en México: inequidad, pobreza y violencia. Nueva Época, LIX(220), 117-146.

Monastiriotis, V. (2009). Examining the Consistency of Spatial Association Patterns Across Socio-economic Indicators: an Application to the Greek Regions. Empirical Economics, 37(1), 25-49.

Mora, F., Reguera, M. y Calvario, J. (2014). Muertes de mujeres y violencia social en tres entidades de la frontera norte de México. Región y Sociedad, (número especial 4), 43-76.

Moran, P. (1950). Notes on Continuous Stochastic Phenomena. Biometrika, 37(1), 17-23.

Moreno, Á. (2005). Impacto de Transmilenio en el crimen de la Avenida Caracas y sus vecindades. Documento CEDE, 55. Bogotá, Colombia: Universidad de los Andes.

Moreno, R. y Vayá, E. (2000). Técnicas Econométricas para el tratamiento de datos espaciales: La econometría espacial. UB 44 manuals. Barcelona, España: Edicions Universitat de Barcelona. 
Moreno, R. y Vayá, E. (2004). Econometría espacial: nuevas técnicas para el análisis regional. Una aplicación a las regiones europeas. Investigaciones regionales, 13(32), 83-106.

Murray, A., McGuffog, I., Western, J. \& Mullins, P. (2001). Exploratory Spatial Data Analysis Techniques for Examining Urban Crime. British Journal of Criminology, 41(2), 309-329.

Owusu, K. \& Owens, C. (2009). Monitoring County-level Chlamydia Incidence in Texas, 2004 - 2005: Application of Empirical Bayesian Smoothing and Exploratory Spatial Data Analysis (ESDA) Methods. International Journal of Health Geographics, 8(12), 1-6.

Pérez, E. y Santos, C. (2011). Diferenciación socio-espacial en la Zona Metropolitana de la Ciudad de México. Investigaciones Geográficas, (74), 92-106.

Ravelo, P. y Sánchez, S. (2006). Resistencia individual y colectiva ante la violencia de género. La experiencia de las obreras de las maquiladoras de Ciudad Juárez. La Ventana, (24), 380-404.

Rodríguez, R. (2014). La política de prevención de violencia de género en México: revisión de las acciones orientadas a los hombres. Alegatos, (88), 691-710.

Rooney, J., Heverin, M., Vajda, A., Crampsie, A., Tobin, K., Byrne, S., Staines, A. \& Hardiman, O. (2014). An Exploratory Spatial Analysis of ALS Incidence in Ireland over 17.5 Years (1995 - July 2013). PLOS ONE, 9(5). [DOI: 10.1371/journal.pone.0096556].

Rupasingha, A. \& Goetz, S. (2007). Social and Political Forces as Determinants of Poverty: a Spatial Analysis. The Journal of Socio-Economics, 36(4), 650-671.

Sánchez, S., Ravelo, P. y Melgoza, J. (2015). Violencia en la ciudad, en el trabajo maquilador y la subjetividad de obreras y obreros en Ciudad Juárez. El Cotidiano, (191), 87-96.

Szonyi, B., Srinath, I., Esteve-Gassent, M., Lupiani, B. \& Ivanek, R. (2015). Exploratory Spatial Analysis of Lyme Disease in Texas - What Can We Learn from the Reported Cases? BMC Public Health, 15(924). [DOI: 10.1186/ s12889-015-2286-0].

Tepechín, A. (2011). Género en contextos de pobreza. México D.F., México: El Colegio de México.

Tobler, W. (1970). A Computer Movie Simulating Urban Growth in the Detroit Region. Economic Geography, 46(Supplement), 234-240.

Tortosa, J. (2009). Feminización de la pobreza y perspectiva de género. Revista Internacional de Organizaciones, (3), 71-89.

Valdivia, M. (2012). Análisis espacial de la dinámica del homicidio de mujeres en México a nivel municipal (2001-2010) Identificación y explicación de patrones de convergencia y polarización territorial. Recuperado de http:// laboratorioespacial.crim.unam.mx/albano/sites/default/files/Estudio\%20 Espacial\%20Violencia\%20UNAM.pdf

Vega, G. (2012). Expresiones de violencia de género en la frontera norte de México: el caso de Ciudad Juárez. Norteamérica, 7(2), 253-284.

Vilalta, C. (2008). Comentarios y mediciones sobre la segregación espacial en la Ciudad de México. Estudios Demográficos y Urbanos, 23(2), 375-413.

Vizcarra, I. (2008). Entre las desigualdades de género: un lugar para las mujeres pobres en la seguridad alimentaria y el combate al hambre. Argumentos, Nueva Época, 21(57), 141-170. 